- The adenomatoid odontogenic tumour (AOT) is a rare tumour of the jaws with completely benign behaviour.

- Treatment of this lesion is via surgical curettage and recurrence seldom if ever occurs. It is ironic that it has been advocated to extract teeth associated with an AOT despite this fact.

- Extraction of the permanent dentition in adolescents is not always in the best interests of the patient especially if it is aesthetically or functionally strategic.

- This study describes the treatment of an adolescent girl for an impacted mandibular canine associated with an AOT.

\title{
Salvage of an impacted canine associated with an adenomatoid odontogenic tumour: A case report
}

\author{
M. H. K. Motamedi, ${ }^{1}$ H. A. Shafeie ${ }^{2}$ and T. Azizi ${ }^{3}$
}

The adenomatoid odontogenic tumour (AOT) has been known by a number of descriptive names (adenoameloblastoma, ameloblastic adenomatoid tumour, glandular ameloblastoma, and adenomatoid ameloblastoma) since it was first reported and later recognised as a distinct odontogenic lesion unrelated to ameloblastoma. Although it was considered to be a variant of ameloblastoma at one time leading surgeons to perform unduly aggressive surgery, the treatment outcome experience has borne out the benign, nonaggressive nature of this lesion. The AOT is now considered to be a hamartoma with completely benign behaviour. Recurrence seldom if ever occurs after surgical curettage. ${ }^{1-4}$ Thus, it appears needless to extract involved anterior teeth associated with the tumour, especially in children. We report a case in which surgical and orthodontic treatment helped to salvage an impacted mandibular canine associated with an AOT in an adolescent girl. We have not found such a procedure to have been done for this tumour in the literature.

\section{CASE REPORT}

A 13-year-old female presented on 7 September 2001 with a small swelling in the vestibular area of the left mandibular canine region. She had no nerve deficit or adenopathy in the face or neck. An orthopantomogram revealed an impacted canine tooth with a relatively-defined radiolucent lesion on the mesial aspect of the length of the crown and root (Fig. 1). The medical history was insignificant and the patient was in good general health. Aspiration of the lesion was negative for fluid. The patient underwent an excisional biopsy

\footnotetext{
1*Associate Professor of Oral and Maxillofacial Surgery, Clinic of Oral and Maxillofacial Surgery and Trauma Research Center, Baqiyatallah University of Medical Sciences / Attending Surgeon, Department of OMFS and Implantology, Azad University of Medical Sciences, Tehran, IR Iran; ${ }^{2}$ Assistant Professor of Orthodontics, Department of Orthodontics, Shahid Beheshti University of Medical Sciences, Tehran, IR Iran; ${ }^{3}$ Assistant Professor of Oral and Maxillofacial Pathology, Baqiyatallah University of Medical Sciences, Tehran, IR Iran. *Correspondence to: Dr Mohammad Motamedi, Africa Expressway, Golestan Street, Giti Blvd. No. 11 Tehran, 19667, IR Iran

Email:motamedical@lycos.com
}

\section{Refereed Paper}

Received 13.09.04: Accepted 15.02.05

doi: $10.1038 /$ sj.bdj.4812522

๑ British Dental Journal 2005; 199: 89-90 under local anaesthesia. A trapezoid flap was reflected from mesial and distal aspects of the involved tooth. The lesion was completely removed after separating the capsule from the bone and incising it off the canine tooth surface. Minimal bone removal in the bed of the lesion was done with a rose bur. Clinically, nothing was left attached to the canine tooth surface. The apex of the root was not involved by the lesion. The wound was irrigated and the flap was sewn apically leaving the crown exposed for bracket bonding. After biopsy results confirmed an AOT (Fig. 2) it was decided not to remove the tooth in view of the benign nature of the lesion and rare chance of a recurrence. Orthodontic treatment was started two weeks postoperatively. The canine was brought into occlusion and the patient is doing well now almost four years postoperatively (Fig. 3).

\section{DISCUSSION}

The adenomatoid odontogenic tumour is usually encountered during the second decade; however, reported cases range in age between four and 80 years. There is a slightly female over male incidence, an almost 2:1 site preference for maxilla over mandible, and a marked predilection for the anterior jaws (over 85\% of lesions appear anterior to the second premolar teeth). ${ }^{1-5}$ The AOT has several radiographic appearances that may be considered typical but not pathognomonic. The lesion frequently appears as a well-circumscribed, pear-shaped radiolucency (stem portion toward the alveolar surface) lying between divergent roots of the canine and first premolar teeth (or between incisors or premolars); or as a unilocular, radiolucent lesion that is well circumscribed or bounded by a radiopaque margin, lateral to or containing an impacted tooth (similar to a follicular or dentigerous cyst). Frequently, small, variably shaped faint or marked radiopaque particles may be distributed throughout the lesional space or are concentrated within a small area of a predominantly radiolucent lesion. Rarely, some lesions appear heavily calcified. ${ }^{1-5}$

\section{Histopathology}

Histologically, the lesion is usually partially cystic and surrounded by a well-developed fibrous connective tissue capsule. The luminal surface is lined by a reduced odontogenic epithelium that leads to thickened mural proliferations of the AOT, which project into and partially or almost completely 


\section{PRACTICE}

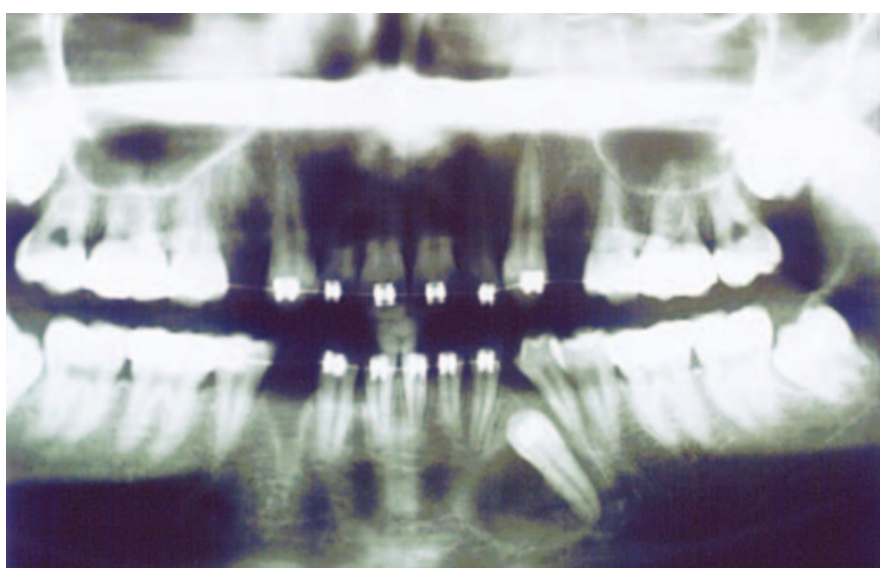

Fig. 1 Panoramic radiograph of the patient revealing an impacted canine tooth with a relatively-defined radiolucent lesion on the mesial aspect of the length of the crown and root.

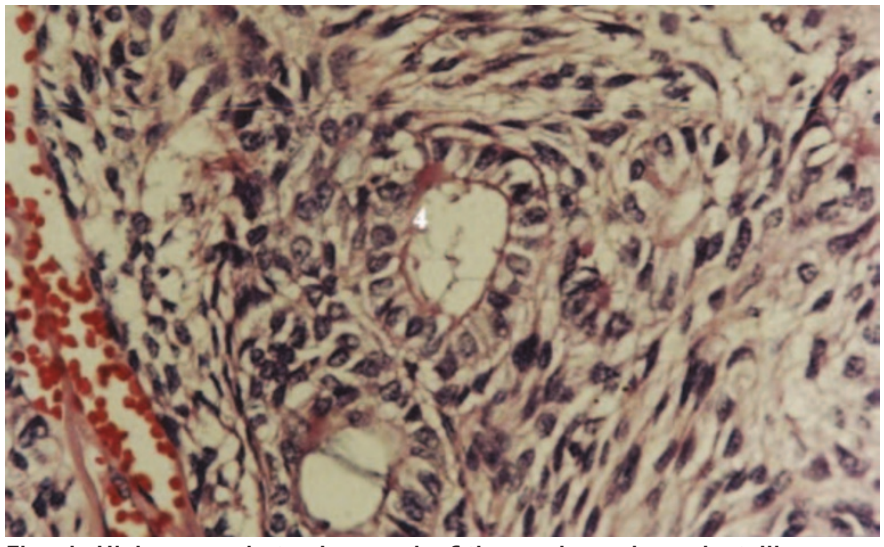

Fig. $2 b$ High power photomicrograph of the specimen shows duct-like epithelial structures with the nuclei of the columnar cells polarised away from the central space (HCE x 400).

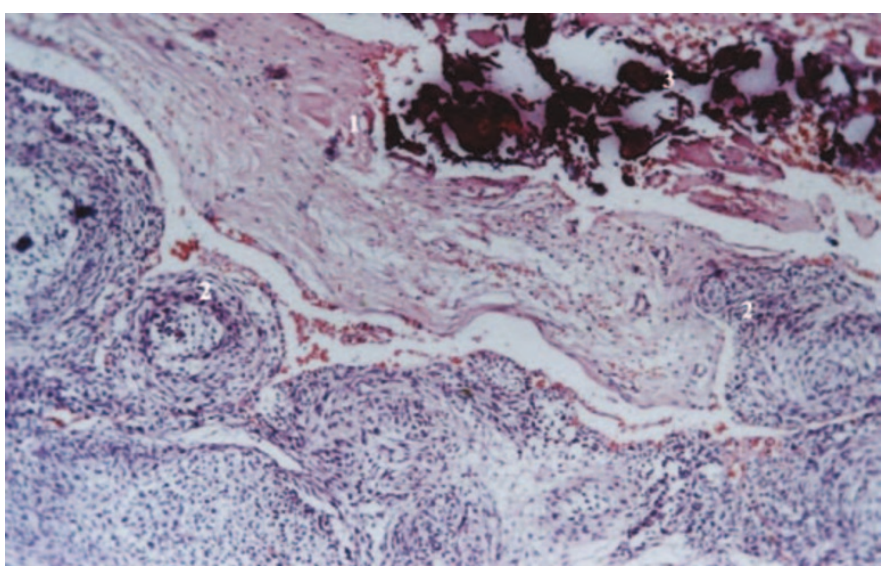

Fig. 2a Low power photomicrograph of the specimen shows thick fibrous capsule surrounding the tumour which is composed of rosettes of spindle shaped epithelial cells. (H\&tE x 250).

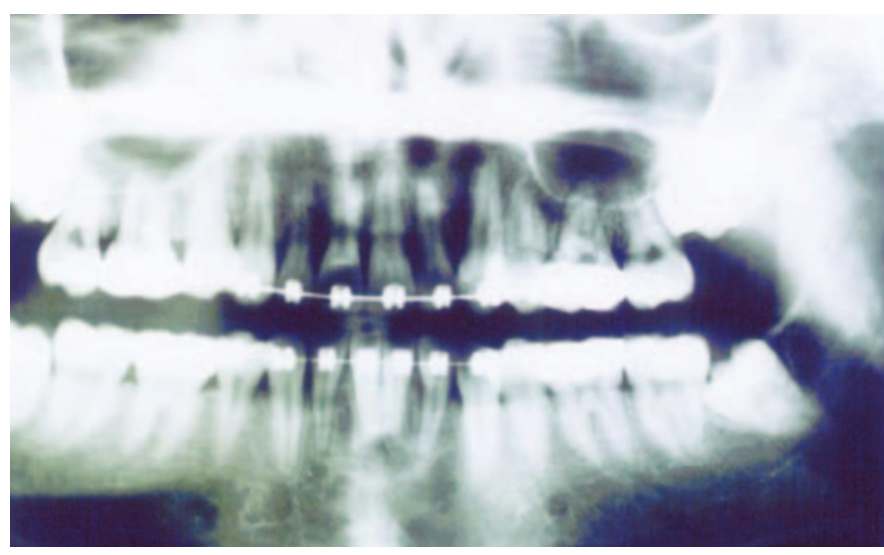

Fig. 3. Panoramic radiograph of the patient three years postoperatively. The canine tooth has been brought into occlusion and the lesion has completely filled with bone. fill a cystic lumen with tumour lobules. The characteristic AOT lesional tissue is composed of a profuse complex of rounded, rosette-like and stream-like aggregates of spindle-shaped odontogenic epithelial cells, similar in size and shape to the stratum intermedium cells of the developing tooth germ (Fig. 2a). Within the epithelial masses, some of the rounded and rosette forms appear to become hollow, to take on ductlike shapes, and to be lined with a single layer of polarised cuboidal or columnar epithelium cells giving it the adenomatoid appearance (Fig. 2b). Frequently, eosinophilic droplets (likened to enameloid products) are seen within the tubes or lying among the spindled epithelial masses. To a greater or lesser degree, calcifications occur within the lumina of the ducts, scattered among the epithelial masses or in the stroma; occasional calcifications have a cementum- or dentine-like appearance. ${ }^{1-5}$

\section{Management}

Due to site, age, and imaging characteristics, lesions such as periodontal cyst, dentiger- ous cyst, calcifying odontogenic cyst (COC), calcifying epithelial odontogenic tumour (CEOT), or desmoplastic ameloblastoma can be considered in the differential diagnosis. If sufficient suspicion exists that the lesion could be a CEOT or desmoplastic ameloblastoma, one or several biopsies of the lesion should be performed, since treatment varies. The accumulated world experience with AOT, as reflected in 30 or more years of reports, depicts a lesion in which conservative management produces a uniformly excellent outcome without recurrence. In almost all instances, the lesion may be removed by surgical enucleation. After reflection of an ample mucosal flap and widened entrance through the usually thinned and expanded cortical bone, the connective tissue capsule of the lesion is encountered. Enucleation is achieved by separation of the lesion from bone without perforating the capsule. Portions of the capsule may be thinned or inflamed, permitting fragments of the lesion to escape the connective tissue envelope.
Inspection, irrigation, and gentle curettage of the resultant cavity remove any residual lesion. Primary closure of the wound can be performed. ${ }^{1-4}$ We feel, however, that impacted teeth incorporated within the lesion need not be removed uniformly, and teeth whose radicular bone cover has been resorbed by the lesion should be endodontically treated.

1. Gold L. Odontogenic tumours: Surgical pathology and management: In Fonseca R J Oral and

Maxillofacial Surgery. pp 328-330. Philadelphia: WB Saunders, 2000

2. Assael L. Surgical management of odontogenic cysts and tumours. In Peterson L J et al. Principles of oral and maxillofacial surgery. Vol. 3. pp 698-701. UK: Lippincott, 1992

3. Waldron $\mathrm{CA}$ : Odontogenic cysts and tumours In Neville B W, Damm D D, Allen C M, Bouquot J E. Oral and maxillofacial pathology. pp 529-531. Philadelphia: WB Saunders, 1995.

4. Wood N K, Goaz P W, Jaocobs M C. Bony lesions. In Wood N K, Goaz P W. Differential diagnosis of oral and maxillofacial lesions. pp 289-290. Philadelphia: CV Mosby, 1997.

5. Philipsen H P, Reichart P A. Adenomatoid odontogenic tumour: facts and figures. Oral Oncol 1999; 35: 125-131. 